\title{
Management of Hyperacute Stroke during the Coronavirus Disease of 2019 Pandemic: The Modified Code Stroke in a Medical Center in Taiwan
}

\author{
Po-Yu Lin, ${ }^{\mathrm{a}}$ Yu-Ming Chang, ${ }^{\text {a }}$ Chih-Yuan Huang, ${ }^{\mathrm{b}}$ Chih-Hao Lin, ${ }^{\mathrm{c}}$ Ming-Tsung Chuang, ${ }^{\mathrm{d}}$ Po-Lin Chen, \\ Pi-Shan Sung, ${ }^{\text {a }}$ Chih-Hung Chen ${ }^{a}$ \\ aDepartment of Neurology, National Cheng Kung University Hospital, College of Medicine, National Cheng Kung University, Tainan, Taiwan \\ ${ }^{b}$ Division of Neurosurgery, Department of Surgery, National Cheng Kung University Hospital, College of Medicine, National Cheng Kung \\ University, Tainan, Taiwan \\ 'Department of Emergency Medicine, National Cheng Kung University Hospital, College of Medicine, National Cheng Kung University, Tainan, \\ Taiwan \\ dDepartment of Medical Imaging, National Cheng Kung University Hospital, College of Medicine, National Cheng Kung University, Tainan, \\ Taiwan \\ eCenter for Infection Control and Department of Internal Medicine, National Cheng Kung University Hospital, College of Medicine, National \\ Cheng Kung University, Tainan, Taiwan
}

\section{Dear Sir:}

The coronavirus disease of 2019 (COVID-19) pandemic has caused significant stress to the healthcare practice. Ischemic stroke was observed in 2.3\% hospitalized COVID-19 patients and $4.5 \%$ patients with severe COVID-19 infections. ${ }^{1}$ In this letter, we report a "modified code stroke" in the management of hyperacute stroke under the COVID-19 crisis followed in a tertiary medical center in Taiwan.

The Taiwan Central Epidemic Command Center (CECC) has coordinated nationwide preventive strategies to intercept the spread of COVID-19. Besides laboratory confirmed COVID-19 cases, the CECC has defined three risk levels for COVID-19 infection. ${ }^{2}$ People having unprotected contact with confirmed COVID-19 patients or travelling from abroad within the past 14 days are either home-isolated or quarantined and tested for severe acute respiratory syndrome coronavirus 2 (SARS-CoV-2), if symptoms developed. Patients with pneumonia, and highrisk social status (medical workers, pulblic transport workers, history of joining public gathering) with symptoms of fever, respiratory tract infection, anosmia, and/or dysgeusia are tested for SARS-CoV-2 as community-based surveillance. Till April 18, 2020, 52,445 SARS-CoV-2 reverse-transcription polymerasechain reaction (RT-PCR) tests were performed in Taiwan, of which 343 were confirmed COVID-19 cases, 333 were included in the 14,151 tests of patients at airports or under home isolation/quarantine (positive rate $2.3 \%$ ), and 10 were included in the 38,294 tests of community-based surveillance (positive rate $0.026 \%$ ). The risk of community transmission was considered low.

The National Cheng Kung University Hospital (NCKUH) is a 1,320-bed tertiary medical center in Southern Taiwan. Figure 1 summarizes the "modified code stroke" followed in the NCKUH, and the strategies are summarized in Supplementary Table 1. Patients used to be treated using the standard code stroke (Supplementary methods ${ }^{3-5}$ ) are treated using the "modified code stroke" and transported to the negative-pressure isolation room if their clinical infection screen based on exposure history and/or symptoms result in positive or poor-quality. All medical personnel in close proximity with the patients wear personal protective equipment to prevent air, droplet, and/or contact infection. ${ }^{6}$ The patients wear surgical masks throughout the "modified code stroke."

We use the modified National Institutes of Health Stroke Scale (NIHSS) to evaluate the severity of stroke. To avoid the removal of patients' surgical masks, we score 1 point for facial palsy if the patient has mild dysarthria, and 2 points if the dysarthria is moderate to severe. The finger-nose-finger test is 


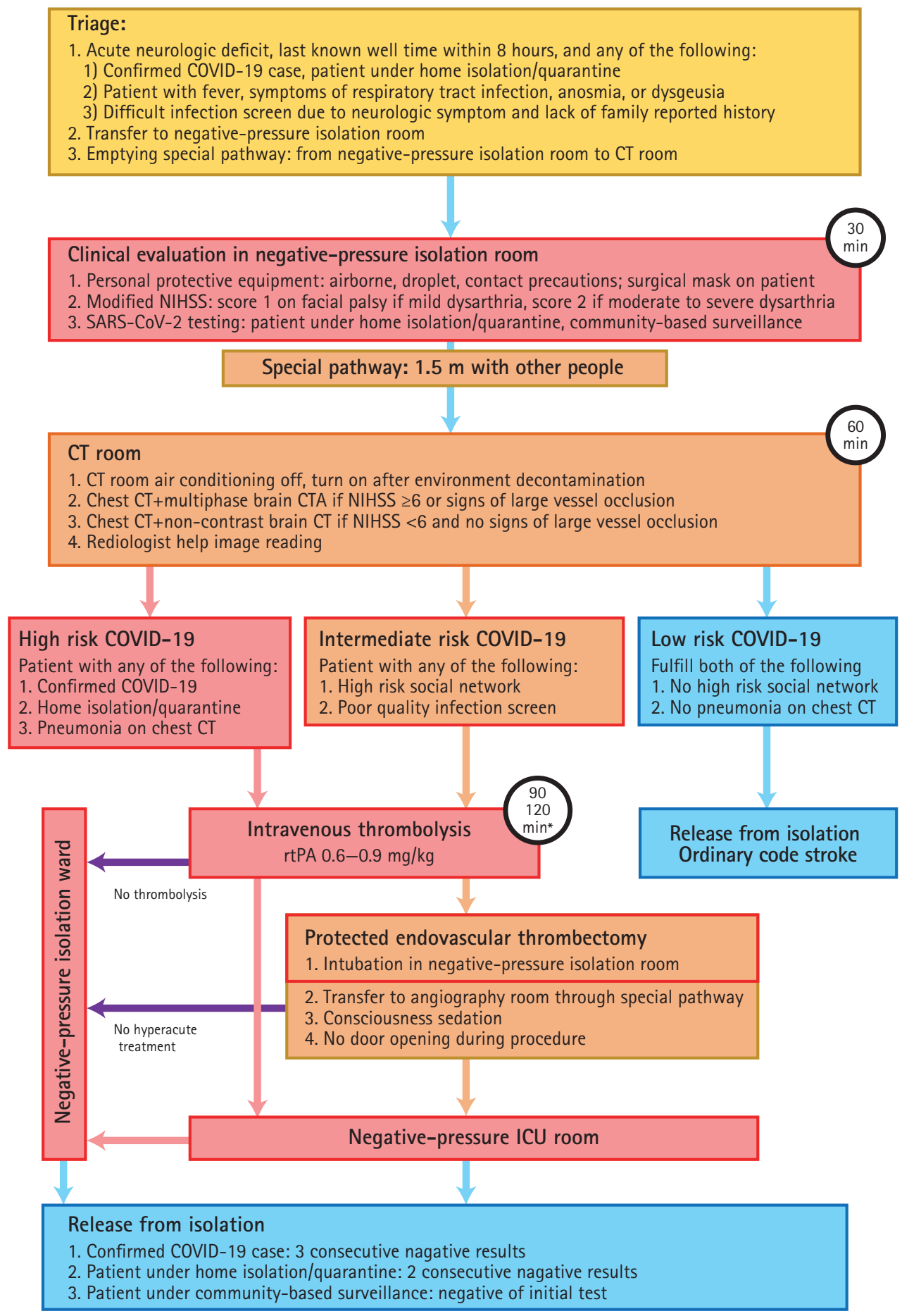

Figure 1. Modified stroke protocol in the National Cheng Kung University Hospital, Taiwan. The box with red rim indicates negative-pressure isolation environment. The box with orange rim indicates environments with modifications in ventilation. The box with yellow rim represents medical staff wearing personal protective equipment. The goal is achieve a door-to-needle time for intravenous thrombolysis as 90 minutes, if non-contrast brain computed tomography (CT) is performed and 120 minutes, if multiphase CT angiography is performed. COVID-19, coronavirus disease of 2019; CTA, computed tomography angiography; NIHSS, National Institutes of Health Stroke Scale; SARS-CoV-2, severe acute respiratory syndrome coronavirus 2; rtPA, recombinant tissue plasminogen activator; ICU, intensive care unit. *90 minutes if non-contrast CT, 120 minutes if multiphase CTA.

substituted with the finger-to-nose test to decrease contact exposure. Before neurological evaluation, a nasopharyngeal swab is obtained early from the patient and screened for SARSCoV-2 by RT-PCR, to promote hemostasis of the swabbed site 
before potential thrombolysis treatment. While obtaining the nasopharyngeal swab, the patient's surgical mask is displaced inferiorly to expose the nostril, but it covered the patient's mouth to minimize the possibility of droplet spread.

After clinical evaluation, the patient is transported to the computed tomography (CT) room through a special corridor that ensures a distance of at least $1.5 \mathrm{~m}$ between the patient and other people. The air conditioning of the CT room is turned off throughout the examination and is turned on after surface decontamination and disinfection with ultraviolet light. Noncontrast chest CT is performed to evaluate the presence of pneumonia. The choice of brain scan depends on the potential need for endovascular thrombectomy (EVT), determined clinically by NIHSS, signs of large vessel occlusion, and risk of COVID-19, as discussed later.

After clinical and radiographic evaluations, we assess the risk of COVID-19. Patients with confirmed COVID-19, under homeisolation or quarantine, or with evidence of pneumonia on chest $\mathrm{CT}$ are considered as high risk of COVID-19. Patients with high-risk social status accompanied by fever and symptoms of respiratory tract infection are considered as intermediate risk of COVID-19. The remaining are considered as low risk of COVID-19. Patients with low risk are released from the isolation to be treated by the standard code stroke. Patients with high or intermediate risk are transferred to the negative-pressure isolation room. The eligibility and protocol of intravenous thrombolysis are same as in standard code stroke.

Taiwan Stroke Society suggested that the risk of infectious exposure in medical workers treating patients with high risk of COVID-19 should be considered ${ }^{7}$ due to limited number of neuro-interventionists and lack of negative-pressure angiography rooms in Taiwan. In NCKUH, we excluded patients with high risk of CIVID-19 from EVT in "modified code stroke."

Patients with intermediate risk of COVID-19 can undergo "protected EVT" if eligible. These patients are intubated in the negative-pressure isolation room, ${ }_{1}^{8}$ and an endotracheal tube is connected to a portable ventilator and closed sputum-suction system. During the procedure, the angiography room is under positive pressure as usual. The patient is under conscious sedation, and ventilation is supported by the portable ventilator to avoid disconnection of the closed respiratory system. After the procedure, the same disinfection procedures as the CT room are performed.

After hyperacute treatment, the patient is admitted to a negative-pressure room or single room in the intensive care unit (ICU). All patients with intravenous thrombolysis and EVT are advised follow-up brain CT 24 hours after treatment. We conduct the SARS-CoV-2 RT-PCR tests every 24 hours. Con- firmed patients with COVID-19 are released from isolation only after three consecutive negative results. Home-isolated/quarantined patients are released after two consecutive negative results. Patient under community-based surveillance are released after negative initial test results. Brain MRI is performed after the patient's release.

This protocol can be further modified in the future. First, EVT in patients with high risk of COVID-19, especially in home-isolated/quarantined patients, can be re-initiated if a reliable, highsensitivity, rapid test of SARS-CoV-2 is developed. Second, training emergency medical technicians in the pre-hospital notification of code stroke can minimize the time required for in-hospital preparation. Third, if all ICU beds are occupied by critical cases, early step-down transfer may be reasonable. ${ }^{9}$ Finally, evidence regarding the influence of COVID-19 on stroke and hyperacute stroke treatment is limited. ${ }^{10}$ Further studies are warranted, and new evidence will further change the "modified code stroke."

\section{Supplementary materials}

Supplementary materials related to this article can be found online at https://doi.org/10.5853/jos.2020.01599.

\section{References}

1. Mao L, Jin H, Wang M, Hu Y, Chen S, He $\mathrm{Q}$, et al. Neurologic manifestations of hospitalized patients with coronavirus disease 2019 in Wuhan, China. JAMA Neurol 2020 Apr 10 [Epub]. https://doi.org/10.1001/jamaneurol.2020.1127.

2. Taiwan Central Epidemic Command Center. CECC measures for following up on persons at risk of infection. Taiwan Centers for Disease Control. https://www.cdc.gov.tw/En/File/Get/ Bj-bAcCOSN3kVWwG1gKWxA. 2020. Accessed May 14, 2020.

3. Tang SC, Tsai LK, Chen CJ, Lee CW, Wang KC, Lai YJ, et al. 2019 Taiwan Stroke Society guideline for endovascular thrombectomy in acute ischemic stroke patients. Formos J Stroke 2019;1:77-89.

4. Chen $\mathrm{CH}_{1}$ Hsieh HC, Sung SF, Hsieh CY, Chen PL, Tsai LK, et al. 2019 Taiwan Stroke Society guideline for intravenous thrombolysis in acute ischemic stroke patients. Formos J Stroke 2019;1:1-22.

5. Powers WJ, Rabinstein AA, Ackerson T, Adeoye OM, Bambakidis NC, Becker K, et al. Guidelines for the early management of patients with acute ischemic stroke: 2019 update to the 2018 guidelines for the early management of acute ischemic stroke: a guideline for healthcare professionals from the American Heart Association/American Stroke Associa- 
tion. Stroke 2019;50:e344-e418.

6. Taiwan Centers for Disease Control. Interim guidelines for clinical management of SARS-CoV-2 Infection (5th edition). Taiwan Centers for Disease Control. https://www.cdc.gov.tw/ En/File/Get/_Dv_q75ZjLcNeRvInrPgUg. 2020. Accessed May 14, 2020.

7. Chen $\mathrm{CH}$, Liu CH, Sung PS, Hsieh CY. Taiwan Stroke Society recommendations for hyperacute stroke management during the COVID-19 pandemic. Formos J Stroke 2020;2:1-4.

8. Fraser JF, Arthur AS, Chen M, Levitt M, Mocco J, Albuquerque FC, et al. Society of Neurolnterventional Surgery recommendations for the care of emergent neurointerventional patients in the setting of covid-19. J Neurointerv Surg 2020 Apr 15 [Epub]. https://doi.org/10.1136/neurintsurg-2020-016098.

9. Temporary emergency guidance to US stroke centers during the COVID-19 pandemic. Stroke 2020 Apr 1 [Epub]. https:// doi.org/10.1161/STROKEAHA.120.030023.
10. Oxley TJ, Mocco J, Majidi S, Kellner $C P$, Shoirah $H$, Singh IP, et al. Large-vessel stroke as a presenting feature of covid-19 in the young. N Engl J Med 2020;382:e60.

Correspondence: Pi-Shan Sung

Department of Neurology, National Cheng Kung University Hospital, College of Medicine, National Cheng Kung University, No.138, Sheng Li Road, Tainan City 704, Taiwan

Tel: +886-6-2353535-5481

Fax: +886-6-2374285

E-mail: pishansung@gmail.com

Received: May 6, 2020

Revised: May 6, 2020

Accepted: May 8, 2020

This work received a grant from the National Cheng Kung University Hospital (Grant number: NCKUH-20200062).

We thank the Stroke Center, Center for Infection Control, Department of Emergency Medicine, Department of Medical imaging, and Department of Anesthesiology at the National Cheng Kung University Hospital in helping us to design this modified protocol. We thank the National Cheng Kung University Hospital for funding this work.

The authors have no financial conflicts of interest. 


\section{Supplementary methods}

\section{The method of designing the modified protocol}

\section{Standard code stroke in National Cheng Kung University Hospital}

National Cheng Kung University Hospital is a university-affiliated tertiary medical center in Southern Taiwan, with 1320 beds, including 124 in the intensive care unit (ICU). Six beds are included in the negative-pressure ICU and 10 in the negative-pressure general ward. In 2019, 852 acute ischemic stroke patients were hospitalized, and intravenous thrombolysis was performed in 69. The eligibility for endovascular thrombectomy was evaluated in 255 patients, and 46 patients had undergone endovascular thrombectomy. The case selections for intravenous thrombolysis and endovascular thrombectomy were based on the recommendations of the Taiwan Stroke Society and the 2019 American Heart Association/American Stroke Association guidelines for managing acute ischemic stroke. ${ }^{3-5}$
Achievement of consensus for the "modified code stroke" Since February 2020, we convened meetings of an assembled multidisciplinary team to discuss the code stroke protocol required in response to the coronavirus disease of 2019 crisis. Members of the meetings included neurologists (Drs. C.H. Chen, P.S. Sung, Y.M. Chang, and P.Y. Lin), a radiologist (Dr. M.T. Chuang), a neuro-interventionist (Dr. C.Y. Huang), emergency physicians (Drs. C.H. Lin, H.I. Shih, and C.C. Hsieh), an anesthesiologist (Dr. C.C. Tseng), an infectious disease specialist in center of infection control (Dr. P.L Chen), and other medical staff involved in this protocol. A preliminary protocol was developed by the two co-authors (Drs. P.S. Sung and P.Y. Lin) using the available published data before April 18, 2020. All co-authors contributed in refining the protocol to facilitate its applications in practice based on the limitations of actual clinical settings. The published version was approved in the meeting conducted on April 22, 2020. 
Supplementary Table 1. Summary of strategies in the modified code stroke

Infection control

Medical workers should wear personal protective equipment to prevent air, droplet, and contact infection, when they are in close proximity with a suspected coronavirus disease of 2019 (COVID-19) patient.

A suspected COVID-19 patient should wear a surgical mask during the entire evaluation process of the modified code stroke.

Special corridors should be used for transportation of suspected COVID-19 patients to avoid contact with other people. The frequency of patient transportation should be kept to a minimum.

Triage

Patients with positive clinical infection screening based on exposure history or symptoms should be triaged to the modified code stroke.

Clinical evaluation

The National Institutes of Health Stroke Scale should be modified to decrease the risk of infection. To avoid the removal of patients' surgical masks, facial palsy should be scored based on the severity of dysarthria. The finger-nose-finger test should be substituted with the finger-to-nose test.

A nasopharyngeal swab should be obtained from the patient early during the clinical evaluation to promote hemostasis of the swabbed site before potential thrombolysis treatment.

Radiographs

Chest computed tomography (CT) can help in diagnosing pneumonia, infection evaluation, and risk stratification.

The choice of brain scan modality should be based on the patient's clinical eligibility for endovascular thrombectomy. If the patient is eligible clinically, multiphase CT angiography should be performed. If not, non-contrast CT should be performed.

Turning off the air-conditioning of the CT room during patient examination can help in minimizing the risk of droplet spread.

Patient's risk for COVID-19

Patients with confirmed COVID-19, having contact with confirmed cases, travelling abroad, and with pneumonia should be considered as high risk of COVID-19. Endovascular thrombectomy will not be performed in these patients.

Patients with high-risk social status and symptoms of fever and respiratory tract infections should be considered as intermediate risk of COVID-19. Protected endovascular thrombectomy should be performed in these patients, if eligible.

The remaining patients should be considered as low risk of COVID-19. Endovascular thrombectomy should be performed in these patients, if eligible, based on the routine clinical protocol.

Intravenous thrombolysis

The eligibility for intravenous thrombolysis should be based on the usual criteria.

Protected endovascular thrombectomy

The patient should be intubated in a negative-pressure room. The endotracheal tube should be connected to a portable ventilator and closed sputum-suction system.

The patient should be under conscious sedation during the endovascular thrombectomy.

The door of the angiography room should be closed throughout the procedure. The angiography room should have positive-pressure ventilation as usual. 